\title{
Outcome Score Measurement and Clinical Trials for Hip Fracture Patients
}

Miguel A Fernandez ${ }^{1 *}$, Alexander L Aquilina ${ }^{2}$, and Matthew L Costa ${ }^{3}$

$1^{*}$ Mr Miguel A Fernandez MBBS MRCS PhD, Specialist Registrar in Trauma \& Orthopaedic Surgery, University Hospitals Coventry \& Warwickshire NHS Trust, Clifford Bridge Road, Coventry, CV2 2DX, UK. fernandezm@doctors.org.uk, Tel. 07939250006

$2 \mathrm{Mr}$ Alexander L Aquilina BSc MBChB MRCS, Academic Clinical Fellow in Trauma \& Orthopaedic Surgery, University Hospitals Coventry \& Warwickshire NHS Trust, Clifford Bridge Road, Coventry, CV2 2DX, UK. alexaquilina@gmail.com

${ }^{3}$ Professor Matthew L Costa PhD FRCS(Tr \& Orth), Professor of Trauma \& Orthopaedic Surgery, University of Oxford, Kadoorie Centre, John Radcliffe Hospital, Headley Way, Oxford OX3 9DU, UK. Matthew.costa@ndorms.ox.ac.uk

*Corresponding author 


\section{ABSTRACT}

Hip fracture is a global problem both for patients and healthcare systems. Patients with a hip fracture have complex health and social needs which present challenges to measuring outcomes. In this review we discuss how knowledge of what patients consider important in their recovery has increased our understanding of how to measure outcomes in this patient population, the work towards establishing a core set of outcomes, and how exploiting existing infrastructure in the UK has provided a powerful framework within which to undertake research.

Keywords: Hip fracture, Outcome score, Clinical trials

\section{INTRODUCTION}

Hip fracture is an increasing global problem with a projected rise from the 1.31 million $^{1}$ hip fractures in 1990 to 6.26 million by $2050 .{ }^{2}$ The estimated socio-economic costs are substantial; hip fractures represent $0.1 \%$ of the global burden of disease worldwide and $1.4 \%$ in the established market economies. ${ }^{1}$ In 2014 there were 64,102 hip fractures in the UK, occupying over 4000 inpatient beds at any one time. ${ }^{3}$ Patients sustaining a hip fracture often have multiple co-morbidities, poor pre-operative health status, and cognitive impairment. ${ }^{4}$ These factors, coupled with high post injury mortality ${ }^{5}$ and increasingly complex social and rehabilitation needs, ${ }^{6}$ make measuring outcomes in this patient group both a priority and a challenge.

Traditionally, there has been little consensus on outcome assessment after hip fracture and studies have focused on outcomes such as mortality and surgical implant success as well as process related outcomes such as length of hospital stay and time to surgery. ${ }^{7}$ However, with 
the growing recognition of hip fracture as a marker of deteriorating general health status, and the drive to measure patient reported outcomes, there is now more focus on general quality of life outcome tools to capture the broad domains of health considered important by the patients recovering from a hip fracture.

In this article, we summarise the common outcome measures used in hip fracture studies, the advances in our understanding of what patients themselves consider important in their recovery and the progress made in establishing a consensus on a core set of outcome measures to facilitate pooling of data and meta-analysis. Modern clinical trials for hip fracture patients will need to measure outcomes which evaluate both the benefit to the patient and the cost effectiveness of the intervention. This is necessary to establish the high quality evidence required to guide policy and practice for this important and challenging group of patients.

\section{OUTCOMES USED IN HIP FRACTURE STUDIES}

A review of the published literature reveals a number of different outcome scales commonly used in hip fracture studies..$^{7-9}$ Table I summarises the most commonly used scales subdivided into the following groups: mobility, general quality of life, activities of daily living (ADL), and joint specific. Here we provide a brief description of these outcomes.

\section{Mobility}

The Timed-Up-and-Go TUG ${ }^{10}$ test records the time it takes to rise from a chair, walk 3 metres, turn around, and return to a seated position in the chair. It has been shown to be a valid and reliable tool for hospital inpatients rehabilitating from orthopaedic injuries ${ }^{11}$ and has been the most commonly used mobility score in previous hip fracture studies. ${ }^{7}$ Other measures of 
mobility include the timed walk, in which gait velocity is calculated by measuring the time taken to walk 10 metres, and outcomes scales which include a single-item assessment of indoor/outdoor mobility, such as those used in the National Hip Fracture Database (NHFD).

\section{Health Related Quality of Life (HRQOL)}

The EuroQuol 5-Dimension (EQ-5D) ${ }^{12}$ is a generic health-related quality-of-life outcome tool which consists of a visual analogue scale (VAS) for self-rated health on a scale from 'best imaginable health state' to 'worst imaginable health state', and a health status instrument with a five-level response (no problems, slight problems, moderate problems, severe problems and extreme problems) for the following five domains: mobility, self-care, usual activities, pain and discomfort, and anxiety and depression. The EQ-5D can be used to estimate quality adjusted life years (QALYS) as part of a health economic analysis and is responsive to change both when self reported ${ }^{13}$ and when proxy reported for patients with cognitive impairment. ${ }^{14}$ These attributes, together with the low patient and clinician burden (the EQ-5D takes less than 5 minutes to complete), make the EQ-5D one of the tools most suited to measuring outcome in the hip fracture population. ${ }^{13,15}$

The Short Form Health Questionnaire is a 36-item generic patient reported outcome scale which uses eight subscales (physical functioning, the physical role, pain, general health, vitality, social function, emotional function and mental health) which can be clustered into physical and mental component scores. ${ }^{16}$ The SF-36 has proven useful in surveys of general and specific populations, comparing the relative burden of diseases, and in differentiating the health benefits produced by a wide range of different treatments. ${ }^{16}$ The SF-36 has been increasingly used in the fragility hip fracture population and has been shown to be responsive 
to change over time with permanent declines in SF-36 observed following hip fracture. ${ }^{17,18}$ The scale takes approximately 10 minutes to complete.

\section{Activities of Daily Living (ADL)}

The Barthel Index (BI) provides a measure of ability to undertake ADLs using a 10-item scale addressing activities such as feeding, mobility, dressing, continence and bathing. ${ }^{19}$ The index has been widely used in stroke rehabilitation studies where good internal consistency and validity for functional status have been reported. ${ }^{20}$ In hip fracture, retrospective Barthel index score at pre-injury have been shown to be predictors of mobility at 3-months and early mortality. ${ }^{21,22}$

The Functional Independence Measure $\left(\mathrm{FIM}^{\mathrm{TM}}\right)$ is an 18 -item scale composed of 13 -motor tasks and 5-cognitive tasks that was developed in the USA for medical rehabilitation. ${ }^{23}$ The FIM has been shown to be reliable in the hip fracture patients both when patient and proxy reported, ${ }^{24,25}$ however it takes $30-60$ minutes to undertake and some of the 18 dimensions assessed may not be applicable or considered important to patients with a hip fracture. ${ }^{26}$

\section{Joint Specific Scores}

The Oxford Hip Sore (OHS) is a disease and region specific patient reported outcome tool, initially designed to measure outcome following hip arthroplasty for osteoarthritis. ${ }^{27}$ It takes approximately 5-minutes to complete and consists of 12 -items measuring hip pain and function. ${ }^{28}$ These scores are summed to produce a scale from 0-48, with a score from 40-48 indicating satisfactory joint function. Although the OHS was designed specifically for assessment of joint replacement it has been increasingly used for evaluating other 
interventions including hip fractures where it has been shown to correlate strongly with general measures of health such as the EQ-5D. ${ }^{13}$

The Harris Hip Score (HHS) was originally developed for patients with traumatic arthritis of the hip following dislocation or fracture. ${ }^{29}$ It is completed partly by the patient and partly by a clinician and is made up of a scale consisting of 13-items across the domains of pain, function, activity, and range of motion. ${ }^{29}$ The score takes approximately 5 minutes to complete and has a maximum score of 100 points. In patients with a hip fracture it has been shown to correlate with age at injury and be an independent predictor of postoperative complications. ${ }^{30,31}$

\section{WHAT IS IMPORTANT TO PATIENTS RECOVERING FROM A HIP FRACTURE?}

A number of studies have focused on understanding what patients consider important in their recovery from a hip fracture in order to match the domains of health from existing outcome tools and guide the choice of outcome assessment. ${ }^{32-35}$ These studies are predominantly based on semi-structured interviews with patients/carers with a spectrum of pre-fracture mobility and cognition ranging from those who are physically and mentally active to those with cognitive impairment and limited or no mobility. Despite this variation of pre-fracture health status, patients identified similar themes when talking about their recovery from a hip fracture. The following themes were identified: mobility, self-care, pain, mental well-being, fear of falling, and valued day-to-day activities. ${ }^{32}$ Of these, mobility emerged as the dominant theme ${ }^{32}$ together with an increased dependency on others. ${ }^{35}$ Although concerns about ongoing pain did emerge in the rehabilitation period following surgery, pain was not considered as significant a problem at that stage as it was in the peri-operative period when pain was a key issue. ${ }^{32}$ Different themes were more or less important to different people. Patients who were active prior to their fracture described the importance of regaining their 
pre-fracture activity levels. However the majority of hip fracture patients had restricted prefracture mobility and this group described more limited expectations of recovery. Those with the most severe physical and cognitive impairment had difficulty describing their expectations of recovery following hip fracture at all; in fact their expectations were subsumed in their acceptance of an age-related deterioration in their health-related quality of life. ${ }^{32}$

What emerges from these studies is the multi-dimensional impact of sustaining a hip fracture. Hip fracture affects many different aspects of quality of life, physical and emotional, and for many patients the effects are difficult to isolate from the impact of other areas of gradual deteriorating health..$^{32}$ Many of the themes of recovery identified by patients are represented by the broad domains of health included in the generic health-related quality of life outcome tools described above, rather than the more disease or joint specific outcome tools.

\section{HEALTH RELATED QUALITY OF LIFE IN PATIENTS RECOVERING FROM A HIP FRACTURE}

Prospective cohort studies have explored the impact of sustaining a hip fracture by measuring the temporal profiles of Health-related Quality of Life from pre-injury to 1 year post surgery. ${ }^{36,37}$ Figure 1 shows the observed population mean scores (from a UK cohort $^{36}$ ) in EQ5D at baseline (pre-injury), immediately post-injury, 4-weeks, 4-months and 12-months post surgery. Interestingly, patients report an immediate post-injury quality of life akin to death, i.e. a score of zero. This is followed by a relatively rapid rate of recovery until the 4-month point after which gains in Quality of Life plateau. Indeed, for the majority of patients - the older group with multiple co-morbidities - Quality of Life shows an age-related deterioration between 4 and 12 months following a hip fracture. Those patients with cognitive impairment have significantly lower Quality of Life scores at all time points and in patients over 80 years of age there is minimal additional recovery after the 4-week point. This pattern of recovery 
observed in terms of Health-related Quality of Life is also found for the hip specific $\mathrm{OHS}^{36}$ and for walking ability using both the single item measure of indoor/outdoor walking ability ${ }^{36}$ and the FIM. ${ }^{37}$

The temporal variation in Quality of Life reveals the severity of functional impairment and global deterioration suffered by patients with a hip fracture. This reflects what we have learnt from patients' own experiences of recovery from hip fracture as being part of a gradual decline in function and general health.

\section{ESTABLISHING A CORE SET OF OUTCOMES FOR HIP FRACTURE STUDIES}

Heterogeneity in the outcomes collected between hip fracture studies precludes effective evidence synthesis and meta-analysis. To address this problem there is an initiative ${ }^{38-40}$ to standardise outcomes by establishing a "core outcome set", that is, a minimum set of outcomes which all studies report in any particular field of medicine. This will improve the effectiveness of evidence synthesis by reducing the reporting bias of future trials and promoting inclusion of all available studies.

A UK consensus study, which involved patients/carers, multi-disciplinary clinicians and methodologists, supports a core outcome set consisting of mortality, mobility status, residential status and general health-related quality of life. ${ }^{41}$ The consensus process recommended the use of single item measures of mortality and mobility (indoor/outdoor walking status), and the EQ-5D as the most simple and therefore practical method of measuring quality of life in hip fracture patients. ${ }^{41}$ This core outcome set supports the findings of an international expert consensus which, in addition to those outcomes described in the UK study, recommends the collection of the process-related outcomes of length of hospital stay, time to surgery, drug reactions, place of residence, cost of care, readmission rate and 
complications. ${ }^{42}$ In the UK, these process related outcomes are collected routinely as part of the national audit process - the NHFD. ${ }^{43}$

\section{CLINICAL TRIALS IN HIP FRACTURE SURGERY}

Given the burden of disease and the massive impact upon both patients and healthcare systems, establishing a high quality evidence base for hip fracture surgery is a global priority. This is reflected by the formation of collaborative groups such as the International Fragility Fracture Network and International Hip Fracture Research Collaborative (IHFRC), and large international multi-centre randomised controlled trials such as the INSITE (Intramedullary Nail Versus Sliding Hip Screw Inter-Trochanteric Evaluation, Trial ID: NCT01380444) and HEALTH (Hip Fracture Evaluation with ALternatives of Total Hip Arthroplasty versus Hemi-Arthroplasty, Trial ID: NCT00556842). ${ }^{44}$

In the UK a collaboration between the Scientific Committee of the NHFD, the National Institute for Health Research Clinical Research Network (NIHR CRN) Injuries and Emergencies Specialty Group and clinicians from multiple institutions, seeks to exploit the existing NHFD infrastructure to collect patient-reported outcome data based on the consensus derived core outcome set described above. This collaboration has established a large cohort ${ }^{45}$ of hip fracture patients where all patients follow a standardised treatment pathway and are followed up in a standardized fashion which includes the collection of the core outcome dataset. Furthermore, this standardised comprehensive cohort has formed the control arm for a series of multiple embedded randomised controlled trials.. ${ }^{46,47}$ These pragmatic trials aim to evaluate both the benefit to the patient and the cost effectiveness of the interventions to guide practice and policy. 


\section{SUMMARY/CONCLUSIONS}

The heterogeneous nature of the hip fracture population coupled with the high prevalence of cognitive impairment and high mortality make outcome assessment in this patient group a challenge. Appropriate outcome assessment in hip fracture should reflect an understanding of what is important to the patients themselves in their experience of recovery. Semistructured interviews with patients have identified priority domains of health and allowed researches to 'map' these domains onto existing outcome tools; a process which has highlighted the EQ-5D as the most sensitive and valid tool which can feasibly be administered to the whole hip fracture population. This is supported by the consensus work to establish a core set of outcome measures which all future studies should collect as a minimum data set to facilitate evidence synthesis and meta-analysis.

The incorporation of this core outcome set within the national audit framework of the NHFD, means that hip fracture research in the UK is well placed to advance the body of knowledge in treating this important patient group. 


\section{REFERENCES}

1. Johnell O, Kanis JA. An estimate of the worldwide prevalence, mortality and disability associated with hip fracture. Osteoporos Int 2004;15(11):897-902.

2. Cooper C, Campion G, Melton LJ. Hip fractures in the elderly: a world-wide projection. Osteoporos Int 1992;2(6):285-289.

3. No authors listed. National Hip Fracture Database Annual Report 2015. Royal College of Physicians RCP.

4. Seitz DP, Adunuri N, Gill SS, Rochon PA. Prevalence of Dementia and Cognitive Impairment Among Older Adults With Hip Fractures. J Am Med Dir Assoc 2011;12(8):556-564.

5. Roberts SE, Goldacre MJ. Time trends and demography of mortality after fractured neck of femur in an English population, 1968-98: database study. BMJ 2003;327(7418):771-775.

6. Baker PN, Salar O, Ollivere BJ et al. Evolution of the hip fracture population: time to consider the future? A retrospective observational analysis. BMJ Open 2014;4(4):4405.

7. Hutchings L, Fox R, Chesser T. Proximal femoral fractures in the elderly: How are we measuring outcome? Injury 2011;42(11):1205-1213.

8. Hoang-Kim AA, Schemitsch EE, Bhandari MM, Kulkarni AVA, Beaton DD. Outcome assessment in hip fracture: evaluation of the practicality of commonlyused outcomes in hip fracture studies. Arch Orthop Trauma Surg 2011;131(12):1687-1695.

9. Bryant DM, Sanders DW, Coles CP, Petrisor BA, Jeray KJ, Laflamme GY. Selection of outcome measures for patients with hip fracture. Journal of Orthopaedic Trauma 2009;23(6):434-441.

10. Podsiadlo D, Richardson S. The timed 'Up \& Go': a test of basic functional mobility for frail elderly persons. J Am Geriatr Soc 1991;39(2):142-148.

11. Yeung TSM, Wessel J, Stratford P, MacDermid J. Reliability, Validity, and Responsiveness of the Lower Extremity Functional Scale for Inpatients of an Orthopaedic Rehabilitation Ward. J Orthop Sports Phys Ther 2009;39(6):468-477.

12. Brooks R. EuroQol: the current state of play. Health Policy 1996;37(1):53-72.

13. Parsons N, Griffin XL, Achten J, Costa ML. Outcome assessment after hip fracture: is EQ-5D the answer? Bone and Joint Research 2014;(3):69-75.

14. Bryan S, Hardyman W, Bentham P, Buckley A, Laight A. Proxy completion of EQ-5D in patients with dementia. Qual Life Res 2005;14(1):107-118.

15. Fernandez MA, Griffin XL, Costa ML. Hip fracture surgery: improving the quality 
of the evidence base. The Bone and Joint Journal 2015;97-B(7):875-879.

16. No authors listed. The SF Community - offering information and discussion on health outcomes. www.SF-36.org. (date last accessed 28 January 2016).

17. Ware JE Jr., Gandek B. Overview of the SF-36 Health Survey and the International Quality of Life Assessment (IQOLA) Project. J Clin Epidemiol 1998;51(11):903912.

18. Tidermark J, Bergström G, Svensson 0, Törnkvist H, Ponzer S. Responsiveness of the EuroQol (EQ 5-D) and the SF-36 in elderly patients with displaced femoral neck fractures. Qual Life Res 2003;12(8):1069-1079.

19. MAHONEY FI, BARTHEL DW. FUNCTIONAL EVALUATION: THE BARTHEL INDEX. Md State Med J 1965;14:61-65.

20. Shah S, Vanclay F, Cooper B. Improving the sensitivity of the Barthel Index for stroke rehabilitation. J Clin Epidemiol 1989;42(8):703-709.

21. Burgos E, GÓMEZ-ARNAU JI, Diez R, MUÑOZ L, FERNÁNDEZ-GUISASOLA J, GARCIA DEL VALLE S. Predictive value of six risk scores for outcome after surgical repair of hip fracture in elderly patients. Acta Anaesthesiologica Scandinavica 2007;52(1):125-131.

22. Elliott J, Beringer T, Kee F, Marsh D, Willis C, Stevenson M. Predicting survival after treatment for fracture of the proximal femur and the effect of delays to surgery. J Clin Epidemiol 2003;56(8):788-795.

23. No authors listed. Rehab Measures: FIM instrument; trademark of the Uniform Data System for Medical Rehabilitation, a division of UB FOundation Activities, Inc. www.rehabmeasures.org. (date last accessed 29 January 2016).

24. Jones CA, Feeny DH. Agreement Between Patient and Proxy Responses During Recovery After Hip Fracture: Evidence for the FIM Instrument. Archives of Physical Medicine and Rehabilitation 2006;87(10):1382-1387.

25. Petrella RJ, Overend T, Chesworth B. FIM ${ }^{\mathrm{TM}}$ After Hip Fracture: Is Telephone Administration Valid and Sensitive to Change? American Journal of Physical Medicine \& Rehabilitation 2002;81(9):639.

26. Latham NK, Jette DU, Warren RL, Wirtalla C. Pattern of Functional Change During Rehabilitation of Patients With Hip Fracture. Archives of Physical Medicine and Rehabilitation Elsevier, 2006;87(1):111-116.

27. Dawson J, Fitzpatrick R, Murray D, Carr A. Comparison of measures to assess outcomes in total hip replacement surgery. Qual Health Care 1996;5(2):81-88.

28. No authors listed. Orthopaedic Scores. www.orthopaedicscores.com. (date last accessed 29 January 2016).

29. Harris WH. Traumatic arthritis of the hip after dislocation and acetabular 
fractures: treatment by mold arthroplasty. The Journal of Bone and Joint Surgery 1969.

30. Frihagen F, Grotle M, Madsen JE, Wyller TB, Mowinckel P, Nordsletten L.

Outcome after femoral neck fractures: A comparison of Harris Hip Score, Eq-5d and Barthel Index. Injury Elsevier, 2008;39(10):1147-1156.

31. Frihagen F, Nordsletten L, Madsen JE. Hemiarthroplasty or internal fixation for intracapsular displaced femoral neck fractures: randomised controlled trial. BMJ British Medical Journal Publishing Group, 2007;335(7632):1251-1254.

32. Griffiths F, Mason V, Boardman $\mathbf{F}$ et al. Evaluating recovery following hip fracture: a qualitative interview study of what is important to patients. BMJ Open 2015;5(1):e005406.

33. Zidén L, Scherman MH, Wenestam C-G. The break remains - elderly people's experiences of a hip fracture 1 year after discharge. Disabil Rehabil 2010;32(2):103-113.

34. Olsson L-E, Nyström AE, Karlsson J, Ekman I. Admitted with a hip fracture: patient perceptions of rehabilitation. Journal of Clinical Nursing 2007;16(5):853859.

35. Zidén L, Wenestam C-G, Hansson-Scherman M. A life-breaking event: early experiences of the consequences of a hip fracture for elderly people. Clin Rehabil 2008;22(9):801-811.

36. Griffin XL, Parsons N, Achten J, Fernandez M, Costa ML. Recovery of healthrelated quality of life in a United Kingdom hip fracture population: the Warwick Hip Trauma Evaluation - a prospective cohort study. The Bone and Joint Journal 2015;97-B(3):372-382.

37. Beaupre LA, Jones CA, Johnston DWC, Wilson DM, Majumdar SR. Recovery of function following a hip fracture in geriatric ambulatory persons living in nursing homes: prospective cohort study. J Am Geriatr Soc 2012;60(7):1268-1273.

38. Koroshetz W. A core set of trial outcomes for every medical discipline? BMJ 2015;350:h85.

39. Tugwell P, Boers M, Brooks P, Simon L, Strand V, Idzerda L. OMERACT: an international initiative to improve outcome measurement in rheumatology. Trials 2007;8:38-38.

40. No authors listed. Core Outcome Measures in Effectiveness Trials. http://www.comet-initiative.org, (date last accessed 8 January 2016).

41. Haywood KL, Griffin XL, Achten J, Costa ML. Developing a core outcome set for hip fracture trials. The Bone and Joint Journal 2014;96-B(8):1016-1023.

42. Liem IS, Kammerlander C, Suhm N, Blauth M, Roth T, Gosch M, et al. Identifying a standard set of outcome parameters for the evaluation of 
orthogeriatric co-management for hip fractures. Injury 2013;44(11):1403-1412.

43. No authors listed. The National Hip Fracture Database. http://www.nhfd.co.uk, (date last accessed 28 January 2015).

44. Bhandari M, Devereaux PJ, Einhorn TA et al. Hip fracture evaluation with alternatives of total hip arthroplasty versus hemiarthroplasty (HEALTH): protocol for a multicentre randomised trial. BMJ Open 2015;5(2):e006263-e006263.

45. Griffin XL, Achten J, Parsons N, Boardman F, Griffiths F, Costa ML. The Warwick Hip Trauma Evaluation -an abridged protocol for the WHiTE Study: A multiple embedded randomised controlled trial cohort study. Bone and Joint Research 2012;1(11):310-314.

46. Griffin XL, McArthur J, Achten J, Parsons N, Costa ML. The Warwick Hip Trauma Evaluation One -an abridged protocol for the WHiTE One Study: An embedded randomised trial comparing the X-bolt with slidinghip screw fixation in extracapsular hip fractures. Bone and Joint Research 2013;2(10):206-209.

47. Griffin XL, McArthur J, Achten J, Parsons N, Costa ML. The Warwick Hip Trauma Evaluation Two -an abridged protocol for the WHiTE Two Study: An embedded randomised trial comparing the Dual-Mobility withpolyethylene cups in hip arthroplasty for fracture. Bone and Joint Research 2013;2(10):210-213. 